\title{
Trapezoidal approximations of fuzzy numbers with restrictions on the support and core
}

\author{
Przemysław Grzegorzewski ${ }^{*, 1,2}$ Karolina Pasternak-Winiarska ${ }^{2}$ \\ ${ }^{1}$ Systems Research Institute, Polish Academy of Sciences, \\ Newelska 6, 01-447 Warsaw, Poland, \\ ${ }^{2}$ Faculty of Mathematics and Information Science, \\ Warsaw University of Technology, \\ Plac Politechniki 1, 00-661 Warsaw, Poland
}

\begin{abstract}
Fuzzy number approximation by trapezoidal fuzzy numbers which preserves the expected interval is discussed. New operators that fulfill additional requirements for the core and support of the fuzzy number are suggested. These supplementary conditions guarantee the proper interpretation of the solution even for very skew original fuzzy numbers.
\end{abstract}

Keywords: fuzzy number, expected interval, support, core, trapezoidal approximation, trapezoidal fuzzy number.

\section{Introduction}

Trapezoidal approximation of fuzzy numbers was considered by many authors (see, e.g. $[1,2,3,4,5$, $8,15,16,17,18,22,23,25,26,27]$. In [17] a list of criteria which trapezoidal approximation operators should possess was formulated. New approaches and modifications of the approximation operators still appear, e.g. trapezoidal approximation under weighed distance were considered by [27, 23, 24]; bisymmetrical weighting was examined in $[19,20]$, etc.

However, this contribution goes back to the original problem of finding the trapezoidal approximation operator preserving the expected interval nearest to the original fuzzy number. Although the solution of that problem is known and practical algorithms for choosing the optimal operator were given (see [16]), for some peculiar fuzzy numbers even these optimal operators may reveal un undesirable behavior. namely, it may happen that even though the solution is mathematically correct, it may loose any natural interpretation or be counterintuitive. Therefore, our goal now is to improve the existing solution by adding two supplementary conditions which protect it against such unwanted cases.

The paper is organized as follows: In Sec. 2 we introduce basic notation. In Sec. 3 we recall the idea of the trapezoidal approximation of fuzzy numbers, discuss possible problems that can appear and indicate the natural way out. Then in Sec. 4 we show

\footnotetext{
*Corresponding author; e-mail: pgrzeg@ibspan.waw.pl.
}

how to find the improved trapezoidal approximation operator.

\section{Basic concepts}

Let $A$ denote a fuzzy number, i.e. such fuzzy subset $A$ of the real line $\mathbb{R}$ with membership function $\mu_{A}$ : $\mathbb{R} \rightarrow[0,1]$ which is (see $[11]$ ):

- normal (i.e. there exist an element $x_{0}$ such that $\left.\mu_{A}\left(x_{0}\right)=1\right)$

- fuzzy convex (i.e. $\mu_{A}\left(\lambda x_{1}+(1-\lambda) x_{2}\right) \geq$ $\left.\mu_{A}\left(x_{1}\right) \wedge \mu_{A}\left(x_{2}\right), \forall x_{1}, x_{2} \in \mathbb{R}, \forall \lambda \in[0,1]\right)$,

- $\mu_{A}$ is upper semicontinuous,

- $\operatorname{supp}(A)$ is bounded, where $\operatorname{supp}(A)=\operatorname{cl}(\{x \in$ $\left.\left.\mathbb{R}: \mu_{A}(x)>0\right\}\right)$, and $c l$ is the closure operator.

A space of all fuzzy numbers will be denoted by $\mathbb{F}(\mathbb{R})$.

It is known that for any fuzzy number $A$ there exist four numbers $a_{1}, a_{2}, a_{3}, a_{4} \in \mathbb{R}$ and two functions $l_{A}, r_{A}: \mathbb{R} \rightarrow[0,1]$, where $l_{A}$ is nondecreasing and $r_{A}$ is nonincreasing, such that we can describe a membership function $\mu_{A}$ in a following manner

$$
\mu_{A}(x)=\left\{\begin{array}{lll}
0 & \text { if } \quad x<a_{1} \\
l_{A}(x) & \text { if } \quad a_{1} \leq x<a_{2} \\
1 & \text { if } a_{2} \leq x \leq a_{3} \\
r_{A}(x) & \text { if } a_{3}<x \leq a_{4} \\
0 & \text { if } a_{4}<x .
\end{array}\right.
$$

Functions $l_{A}$ and $r_{A}$ are called the left side and the right side of a fuzzy number $A$, respectively.

Moreover, let $A_{\alpha}=\left\{x \in \mathbb{R}: \mu_{A}(x) \geq \alpha\right\}, \alpha \in$ $(0,1]$, denote an $\alpha$-cut of a fuzzy number $A$. As it is known, every $\alpha$-cut of a fuzzy number is a closed interval, i.e. $A_{\alpha}=\left[A_{L}(\alpha), A_{U}(\alpha)\right]$, where $A_{L}(\alpha)=$ $\inf \left\{x \in \mathbb{R}: \mu_{A}(x) \geq \alpha\right\}$ and $A_{U}(\alpha)=\sup \{x \in \mathbb{R}:$ $\left.\mu_{A}(x) \geq \alpha\right\}$.

The core of a fuzzy number $A$ is the set of all points that surely belong to $A$, i.e. $\operatorname{core}(A)=\{x \in$ $\left.\mathbb{R}: \mu_{A}(x)=1\right\}=A_{\alpha=1}$.

The expected interval $E I(A)$ of a fuzzy number $A$ is given by (see [12], [21])

$$
\begin{aligned}
E I(A) & =\left[E I_{L}(A), E I_{U}(A)\right] \\
& =\left[\int_{0}^{1} A_{L}(\alpha) d \alpha, \int_{0}^{1} A_{U}(\alpha) d \alpha\right] .
\end{aligned}
$$


The middle point of the expected interval given by

$$
E V(A)=\frac{1}{2}\left(\int_{0}^{1} A_{L}(\alpha) d \alpha+\int_{0}^{1} A_{U}(\alpha) d \alpha\right)
$$

is called the expected value of a fuzzy number and it represents the typical value of the fuzzy number $A$ (see [12], [21]). Sometimes its generalization, called weighted expected value, might be interesting. It is defined as

$$
E V_{q}(A)=(1-q) \int_{0}^{1} A_{L}(\alpha) d \alpha+q \int_{0}^{1} A_{U}(\alpha) d \alpha
$$

where $q \in[0,1]$ (see [13]). Another parameter characterizing the typical value of the magnitude that the fuzzy number $A$ represents is called the value of fuzzy number $A$ and is defined by (see [10])

$$
\operatorname{Val}(A)=\int_{0}^{1} \alpha\left(A_{L}(\alpha)+A_{U}(\alpha)\right) d \alpha
$$

To describe the nonspecifity of a fuzzy number we usually use so-called width of a fuzzy number (see [9]) defined by

$$
\begin{aligned}
w(A) & =\int_{-\infty}^{\infty} \mu_{A}(x) d x \\
& =\int_{0}^{1}\left(A_{U}(\alpha)-A_{L}(\alpha)\right) d \alpha .
\end{aligned}
$$

The next index characterizing the vagueness of fuzzy number $A$, called the ambiguity, is given by (see $[10])$

$$
A m b(A)=\int_{0}^{1} \alpha\left(A_{U}(\alpha)-A_{L}(\alpha)\right) d \alpha .
$$

\section{Trapezoidal approximation}

Suppose that for a certain reason or just for simplicity we want to find a suitable approximation of a fuzzy number under study. It seems that sufficiently effective simplification of the fuzzy number shape can be reached by the piecewise linear curves leading to triangle, trapezoidal or orthogonal membership functions. Since these three mentioned shapes are particular cases of the trapezoidal one, further on we will consider just the trapezoidal approximation of fuzzy numbers. It means that we want to substitute given fuzzy number $A$ by the trapezoidal fuzzy number $T(A)$, i.e. by a fuzzy number with a following membership function

$$
\mu_{T(A)}(x)= \begin{cases}0 & \text { if } x<t_{1}, \\ \frac{x-t_{1}}{t_{2}-t_{1}} & \text { if } t_{1} \leq x<t_{2}, \\ 1 & \text { if } t_{2} \leq x \leq t_{3}, \\ \frac{t_{4}-x}{t_{4}-t_{3}} & \text { if } t_{3}<x \leq t_{4}, \\ 0 & \text { if } t_{4}<x .\end{cases}
$$

Since we can do this in many ways we need some additional constraints which guarantee that our approximation would be reasonable. One of the most natural idea is to construct $T(A)$ that is the closest to the original fuzzy number $A$ with respect to given distance $d$. Sometimes we add additional requirements which warrant that our approximation would possess some desired properties, like preservation fixed parameters or relations, continuity, etc. This problem was considered by many authors (see, e.g. $[1,2,3,4,5,8,15,16,17,18,22,23,25,26,27]$. For example, it was suggested in [17] to consider the nearest trapezoidal approximation operator preserving the expected interval, i.e. the approximation operator $T$ which produces a trapezoidal fuzzy number $T(A)$ that is the closest with respect to distance $d(A, T(A))$, where

$$
\begin{aligned}
d^{2}(A, T(A))= & \int_{0}^{1}\left[A_{L}(\alpha)-T(A)_{L}(\alpha)\right]^{2} d \alpha \\
& +\int_{0}^{1}\left[A_{U}(\alpha)-T(A)_{U}(\alpha)\right]^{2} d \alpha
\end{aligned}
$$

to given original fuzzy number $A$ among all trapezoidal fuzzy numbers having identical expected interval as the original one, i.e. satisfying a following condition

$$
E I(T(A))=E I(A) .
$$

It is worth noting the invariance of the expected interval assures many other properties (for more details we refer the reader to [17] where the broad list of desired requirements that the approximation operator should possess is also given). The research on this operator was continued in [5], [18], [15], [16].

Whatever trapezoidal approximation is considered the goal reduces to finding such real numbers $t_{1} \leq t_{2} \leq t_{3} \leq t_{4}$ that characterize $T(A)=$ $T\left(t_{1}, t_{2}, t_{3}, t_{4}\right)$. It is so because any trapezoidal fuzzy number is completely described by four real numbers that are borders of its support and core. Let us recall the algorithm for computing the nearest trapezoidal approximation preserving the expected interval given in [16].

\section{Algorithm 1}

Step 1. If $A m b(A) \geq \frac{1}{3} w(A)$ then apply operator $T_{1}(A)=T_{1}\left(t_{1}, t_{2}, t_{3}, t_{4}\right)$ given by

$$
\begin{aligned}
& t_{1}=4 \int_{0}^{1} A_{L}(\alpha) d \alpha-6 \int_{0}^{1} \alpha A_{L}(\alpha) d \alpha \\
& t_{2}=-2 \int_{0}^{1} A_{L}(\alpha) d \alpha+6 \int_{0}^{1} \alpha A_{L}(\alpha) d \alpha \\
& t_{3}=-2 \int_{0}^{1} A_{U}(\alpha) d \alpha+6 \int_{0}^{1} \alpha A_{U}(\alpha) d \alpha \\
& t_{4}=4 \int_{0}^{1} A_{U}(\alpha) d \alpha-6 \int_{0}^{1} \alpha A_{U}(\alpha) d \alpha
\end{aligned}
$$

else 
Step 2. if $E V_{\frac{1}{3}}(A) \leq \operatorname{Val}(A) \leq E V_{\frac{2}{3}}(A)$ then apply operator $T_{2}(A)=T_{2}\left(t_{1}, t_{2}, t_{3}, t_{4}\right)$ given by

$$
\begin{aligned}
t_{1}= & 3 \int_{0}^{1} A_{L}(\alpha) d \alpha+\int_{0}^{1} A_{U}(\alpha) d \alpha \\
& -3 \int_{0}^{1} \alpha A_{L}(\alpha) d \alpha-3 \int_{0}^{1} \alpha A_{U}(\alpha) d \alpha \\
t_{2}= & t_{3}=-\int_{0}^{1} A_{L}(\alpha) d \alpha-\int_{0}^{1} A_{U}(\alpha) d \alpha \\
& +3 \int_{0}^{1} \alpha A_{L}(\alpha) d \alpha+3 \int_{0}^{1} \alpha A_{U}(\alpha) d \alpha \\
t_{4}= & \int_{0}^{1} A_{L}(\alpha) d \alpha+3 \int_{0}^{1} A_{U}(\alpha) d \alpha \\
& -3 \int_{0}^{1} \alpha A_{L}(\alpha) d \alpha-3 \int_{0}^{1} \alpha A_{U}(\alpha) d \alpha
\end{aligned}
$$

else

Step 3. if $\operatorname{Val}(A)<E V_{\frac{1}{3}}(A)$ then apply operator $T_{3}(A)=T_{3}\left(t_{1}, t_{2}, t_{3}, t_{4}\right)$ given by

$$
\begin{aligned}
& t_{1}=t_{2}=t_{3}=\int_{0}^{1} A_{L}(\alpha) d \alpha \\
& t_{4}=2 \int_{0}^{1} A_{U}(\alpha) d \alpha-\int_{0}^{1} A_{L}(\alpha) d \alpha
\end{aligned}
$$

else

Step 4. apply operator $T_{4}(A)=T_{4}\left(t_{1}, t_{2}, t_{3}, t_{4}\right)$ given by

$$
\begin{aligned}
& t_{1}=2 \int_{0}^{1} A_{L}(\alpha) d \alpha-\int_{0}^{1} A_{U}(\alpha) d \alpha \\
& t_{2}=t_{3}=t_{4}=\int_{0}^{1} A_{U}(\alpha) d \alpha .
\end{aligned}
$$

Although expressing conditions differently, the equivalent operators are given in [5] and [23]. Therefore, we conclude that we have received four different operators providing the nearest trapezoidal fuzzy number that preserves the expected value of the original fuzzy number, where $T_{1}$ leads to trapezoidal fuzzy number, $T_{2}$ stands for the operator that leads to triangular fuzzy number with two sides, while $T_{3}$ and $T_{4}$ produce triangular fuzzy numbers with the right side only or with the left side only, respectively.

Unfortunately, for very skew membership functions our optimal approximation operators reveal quite unpleasant behavior. Namely, if $\operatorname{Val}(A)<$ $E V_{1 / 3}(A)$ or $\operatorname{Val}(A)>E V_{2 / 3}(A)$, then it may happen that the core of the corresponding operator's output $T_{3}(A)$ or $T_{4}(A)$, respectively, is disjoint with the core of the original fuzzy number $A$. To illustrate such situation let us consider Example 3 given in [15].

\section{Example}

Suppose a fuzzy number $A$ has a membership function

$$
\mu_{A}(x)= \begin{cases}(x+1)^{2} & \text { if }-1 \leq x \leq 0 \\ 1 & \text { if } 0 \leq x \leq 1 \\ \left(\frac{40-x}{39}\right)^{2} & \text { if } 1 \leq x \leq 40 \\ 0 & \text { otherwise }\end{cases}
$$

Hence we compute immediately its $\alpha$-cuts $A_{\alpha}=$ $[\sqrt{\alpha}-1,40-39 \sqrt{\alpha}]$ and we get $\operatorname{Amb}(A)=\frac{9}{2}<$ $\frac{1}{3} w(A)=\frac{43}{9}$ and $\operatorname{Val}(A)=\frac{43}{10}<E V_{\frac{1}{3}}(A)=$ $\frac{40}{9}$, so the nearest trapezoidal approximation is $T_{3}$ which produces the following triangular fuzzy number $T_{3}(A)=T_{3}\left(-\frac{1}{3},-\frac{1}{3},-\frac{1}{3}, \frac{85}{3}\right)$. It is easily seen that $\operatorname{core}(A)=[0,1]$, core $(T(A))=\left\{-\frac{1}{3}\right\}$ and hence $\operatorname{core}(A) \cap \operatorname{core}(T(A))=\emptyset$.

To avoid such undesired situations Abbasbandy and Hajjari [4] considered trapezoidal approximation preserving the core of a fuzzy number. Although this idea sometimes might be useful, some objections also appear. Actually, $\mu_{A}(x)=1$ leads to perfect information that $x$ surely belongs to $A$. If $\mu_{A}(x)$ is close to 1 we say that $x$ rather belongs to $A$. And conversely, $\mu_{A}(x)=0$ shows that $x$ surely does not belong to $A$ (and belongs to $\neg A$ ) which is also a perfect information. Similarly, $x$ such that $\mu_{A}(x)$ is close to 0 is interpreted as a point that rather does not belong to $A$. However, if $\mu_{A}(x)$ is close to 0.5 we dot know how to classify $x$ because it belong to $A$ and to it's completion $\neg A$ more or less with the same degree. Thus, to sum up, degrees of membership both high (close to 1) and low (close to 0) are much more informative than those close to 0.5 and hence there is no reason to favor the core only and to discriminate against the support. Unfortunately, if we fix both the core and support we obtain a naive approximation $T(A)=T\left(a_{1}, a_{2}, a_{3}, a_{4}\right)$ which neglects completely the shape of the arms of the original fuzzy number $A$.

Therefore, to evade this trap we have to weaken slightly requirements for the core and support preservation. However, we should do it without loosing the main idea at the same time. It seems that the right direction was suggested in [14] (see Def. 2), i.e. instead of the core and support preservation the appropriate inclusions would be desirable. Namely, it seems that the sufficiently strong requirement to be satisfied are

$$
\begin{aligned}
\operatorname{core}(A) & \subseteq \operatorname{core}(T(A)) \\
\operatorname{supp}(T(A)) & \subseteq \operatorname{supp}(A)
\end{aligned}
$$

or, using notation adopted above, we may demand for

$$
\begin{aligned}
{\left[a_{2}, a_{3}\right] } & \subseteq\left[t_{2}, t_{3}\right] \\
{\left[t_{1}, t_{4}\right] } & \subseteq\left[a_{1}, a_{4}\right] .
\end{aligned}
$$

These conditions reflect our concern that each point which surely belong to $A$ would also belong 
to $T(A)$ and that each point which surely does not belong to $A$ would not belong to $T(A)$.

\section{Trapezoidal approximation with restrictions on support and core}

It is easily seen that the $\alpha$-cut of $T(A)$ is equal to $\left[t_{1}+\left(t_{2}-t_{1}\right) \alpha, t_{4}-\left(t_{4}-t_{3}\right) \alpha\right]$. Since a trapezoidal fuzzy number is completely described by four real numbers that are borders of its support and core, our goal reduces to finding such real numbers $t_{1} \leq t_{2} \leq t_{3} \leq t_{4}$ that characterize $T(A)=$ $T\left(t_{1}, t_{2}, t_{3}, t_{4}\right)$. Substituting it into (9) and (10) our problem might be expressed as follows:

find $t_{1}, t_{2}, t_{3}, t_{4}$ which minimize

$$
\begin{aligned}
& d^{2}(A, T(A))= \\
& \quad=\int_{0}^{1}\left[A_{L}(\alpha)-\left(t_{1}+\left(t_{2}-t_{1}\right) \alpha\right)\right]^{2} d \alpha \\
& \quad+\int_{0}^{1}\left[A_{U}(\alpha)-\left(t_{4}-\left(t_{4}-t_{3}\right) \alpha\right)\right]^{2} d \alpha
\end{aligned}
$$

with respect to condition that guaranties the expected interval invariance under approximation

$$
\left[\frac{t_{1}+t_{2}}{2}, \frac{t_{3}+t_{4}}{2}\right]=\left[\int_{0}^{1} A_{L}(\alpha) d \alpha, \int_{0}^{1} A_{U}(\alpha) d \alpha\right]
$$

and the restriction (11)-(12) for the support and core

$$
a_{1} \leq t_{1} \leq t_{2} \leq a_{2} \leq a_{3} \leq t_{3} \leq t_{4} \leq a_{4}
$$

We assume that $a_{1}<a_{2}$ and $a_{3}<a_{4}$ because otherwise lineralization of the left or right arm, respectively, has no sense. It is easily seen that our problem can be solved as two separate minimization programs:

Program 1: find $t_{1}$ and $t_{2}$ which minimize

$$
f_{1}(A, T(A))=\int_{0}^{1}\left[A_{L}(\alpha)-\left(t_{1}+\left(t_{2}-t_{1}\right) \alpha\right)\right]^{2} d \alpha
$$

such that

$$
\frac{t_{1}+t_{2}}{2}-E I_{L}(A)=0
$$

where

$$
a_{1} \leq t_{1} \leq t_{2} \leq a_{2} .
$$

Program 2: find $t_{3}$ and $t_{4}$ which minimize

$$
f_{2}(A, T(A))=\int_{0}^{1}\left[A_{U}(\alpha)-\left(t_{4}-\left(t_{4}-t_{3}\right) \alpha\right)\right]^{2} d \alpha
$$

such that

$$
\frac{t_{3}+t_{4}}{2}-E I_{U}(A)=0
$$

where

$$
a_{3} \leq t_{3} \leq t_{4} \leq a_{4} .
$$

To get a solution of Program 1 we apply the Karush-Kuhn-Tucker theorem for the local minimizer of $f_{1}(\mathbf{t})$ subject to $h_{1}(\mathbf{t})=t_{1}+t_{2}-2 E I_{L}(A)=$
0 and $g_{1}(\mathbf{t})=\left[a_{1}-t_{1}, t_{1}-t_{2}, t_{2}-a_{2}\right] \leq 0$. Then there exist the Lagrange multiplier vector $\xi$ and the Karush-Kuhn-Tucker multiplier $\lambda$ such that

$$
\begin{aligned}
D f_{1}(\mathbf{t})+\xi D h_{1}\left(\mathbf{t}^{*}\right)+\lambda^{T} D g_{1}(\mathbf{t}) & =\mathbf{0}^{T}, \\
\lambda^{T} g_{1}(\mathbf{t}) & =0, \\
\lambda & \geq 0 .
\end{aligned}
$$

In our case, after some calculations, we get

$$
\begin{aligned}
& D f_{1}(\mathbf{t})= {\left[\frac{2}{3} t_{1}+\frac{1}{3} t_{2}+2 \int_{0}^{1} \alpha A_{L}(\alpha) d \alpha\right.} \\
&-2 \int_{0}^{1} A_{L}(\alpha) d \alpha, \\
&\left.\frac{1}{3} t_{1}+\frac{2}{3} t_{2}-2 \int_{0}^{1} \alpha A_{L}(\alpha) d \alpha\right] \\
& D h_{1}\left(\mathbf{t}^{*}\right)=[1,1], \\
& D g_{1}(\mathbf{t})=\left[\begin{array}{cc}
-1 & 0 \\
1 & -1 \\
0 & 1
\end{array}\right] \\
& \lambda^{T}=\left[\lambda_{1}, \lambda_{2}, \lambda_{3}\right] .
\end{aligned}
$$

Therefore, we can rewrite the Karush-KuhnTucker conditions in a following way:

$$
\begin{aligned}
\frac{2}{3} t_{1}+\frac{1}{3} t_{2}+2 \int_{0}^{1} \alpha A_{L}(\alpha) d \alpha & \\
-2 \int_{0}^{1} A_{L}(\alpha) d \alpha+\xi-\lambda_{1}+\lambda_{2} & =0 \\
\frac{1}{3} t_{1}+\frac{2}{3} t_{2}-2 \int_{0}^{1} \alpha A_{L}(\alpha) d \alpha & \\
+\xi-\lambda_{2}+\lambda_{3} & =0 \\
t_{1}+t_{2}-2 E I_{L}(A) & =0 \\
\left(a_{1}-t_{1}\right) \lambda_{1} & =0 \\
\left(t_{1}-t_{2}\right) \lambda_{2} & =0 \\
\left(t_{2}-a_{2}\right) \lambda_{3} & =0 \\
\lambda_{1} & \geq 0 \\
\lambda_{2} & \geq 0 \\
\lambda_{3} & \geq 0 .
\end{aligned}
$$

From $(20)$ we get $t_{2}=2 E I_{L}(A)-t_{1}$ and since by (2) $E I_{L}(A)=\int_{0}^{1} A_{L}(\alpha) d \alpha$ we may rewrite first two equations of the aforementioned system in the following way

$$
\begin{aligned}
t_{1}-4 E I_{L}(A)+ & 6 \int_{0}^{1} \alpha A_{L}(\alpha) d \alpha \\
& +3 \xi-3 \lambda_{1}+3 \lambda_{2}=0 \\
-t_{1}+4 E I_{L}(A)- & 6 \int_{0}^{1} \alpha A_{L}(\alpha) d \alpha \\
& +3 \xi-3 \lambda_{2}+3 \lambda_{3}=0
\end{aligned}
$$

Because of inequalities (24)-(26) we have to consider 9 possible situations. Let us firstly assume 
that $\lambda_{1}=\lambda_{2}=\lambda_{3}=0$. Then adding (27) and (28) we get $\xi=0$ and thus we obtain

$$
\begin{aligned}
& t_{1}=4 E I_{L}(A)-6 \int_{0}^{1} \alpha A_{L}(\alpha) d \alpha \\
& t_{2}=6 \int_{0}^{1} \alpha A_{L}(\alpha) d \alpha-2 E I_{L}(A) .
\end{aligned}
$$

Now suppose that $\lambda_{1}>0$ while still $\lambda_{2}=\lambda_{3}=0$. Then by (21)

$$
\begin{aligned}
t_{1} & =a_{1} \\
t_{2} & =2 E I_{L}(A)-a_{1} .
\end{aligned}
$$

However, when substituting this solution into (27) and (28) we obtain

$$
\lambda_{1}=\frac{2}{3} a_{1}-\frac{8}{3} E I_{L}(A)+4 \int_{0}^{1} \alpha A_{L}(\alpha) d \alpha .
$$

Therefore, since $\lambda_{1}>0$, the last solution holds provided

$$
a_{1}>4 E I_{L}(A)-6 \int_{0}^{1} \alpha A_{L}(\alpha) d \alpha .
$$

Let us now consider $\lambda_{2}>0$ and $\lambda_{1}=\lambda_{3}=0$. Then by (22) and (20) we get $t_{1}=t_{2}=E I_{L}(A)$. Substituting this value into (27) and (28) we obtain

$$
\lambda_{2}=E I_{L}(A)-2 \int_{0}^{1} \alpha A_{L}(\alpha) d \alpha .
$$

Since $\lambda_{2}>0$ hence our last solution will exist provided $E I_{L}(A)>2 \int_{0}^{1} \alpha A_{L}(\alpha) d \alpha$. However,

$$
\lambda_{2}=E I_{L}(A)-2 \int_{0}^{1} \alpha A_{L}(\alpha) d \alpha .
$$

Since $\lambda_{2}>0$ hence our last solution will exist provided $E I_{L}(A)>2 \int_{0}^{1} \alpha A_{L}(\alpha) d \alpha$. However,

$$
\begin{aligned}
& E I_{L}(A)-2 \int_{0}^{1} \alpha A_{L}(\alpha) d \alpha \\
= & \int_{0}^{1} A_{L}(\alpha)-2 \int_{0}^{1} \alpha A_{L}(\alpha) d \alpha \\
= & \int_{0}^{1}(1-2 \alpha) A_{L}(\alpha) d \alpha \\
= & \int_{0}^{1 / 2}(1-2 \alpha) A_{L}(\alpha) d \alpha+\int_{1 / 2}^{1}(1-2 \alpha) A_{L}(\alpha) d \alpha \\
= & \int_{0}^{1 / 2}(1-2 \alpha) A_{L}(\alpha) d \alpha \\
& +\int_{0}^{1 / 2}(2 \beta-1) A_{L}(1-\beta) d \beta \\
= & \int_{0}^{1 / 2}(1-2 \alpha)\left[A_{L}(\alpha)-A_{L}(1-\alpha)\right] d \alpha \leq 0
\end{aligned}
$$

which contradicts the assumption that $\lambda_{2}>0$.
Next situation corresponds to $\lambda_{3}>0$ and $\lambda_{1}=$ $\lambda_{2}=0$. Then by (23) and (20) we get

$$
\begin{aligned}
t_{1} & =2 E I_{L}(A)-a_{2} \\
t_{2} & =a_{2} .
\end{aligned}
$$

Substituting, as usual, the suggested solution into (27) and (28) we obtain

$$
\lambda_{3}=-\frac{2}{3} a_{2}-\frac{4}{3} E I_{L}(A)+4 \int_{0}^{1} \alpha A_{L}(\alpha) d \alpha .
$$

Therefore, since $\lambda_{3}>0$, the last solution exists provided

$$
a_{2}<6 \int_{0}^{1} \alpha A_{L}(\alpha) d \alpha-2 E I_{L}(A) .
$$

Now let us consider what happen if $\lambda_{1}>0, \lambda_{2}=0$ and $\lambda_{3}>0$. By (21) and (23) we obtain

$$
\begin{aligned}
& t_{1}=a_{1} \\
& t_{2}=a_{2} .
\end{aligned}
$$

As an immediate conclusion we get that the last solution is possible provided

$$
E I_{L}(A)=\frac{a_{1}+a_{2}}{2} .
$$

Moreover, equations (27) and (28) provide the following formulae

$$
\frac{2}{3} a_{1}-\frac{8}{3} E I_{L}(A)+4 \int_{0}^{1} \alpha A_{L}(\alpha) d \alpha=\lambda_{1}+\lambda_{3}
$$

which, by the assumptions $\lambda_{1}>0$ and $\lambda_{3}>0$ and (31) gives us two requirements for the solution existence

$$
\begin{aligned}
& a_{1}>4 E I_{L}(A)-6 \int_{0}^{1} \alpha A_{L}(\alpha) d \alpha \\
& a_{2}<6 \int_{0}^{1} \alpha A_{L}(\alpha) d \alpha-2 E I_{L}(A) .
\end{aligned}
$$

Next situation to be considered is $\lambda_{1}=0, \lambda_{2}>0$ and $\lambda_{3}>0$ which, by (22) and (23) leads to $t_{1}=$ $t_{2}=a_{2}$. However, such solution is not possible, because it means by $(20)$ that $a_{2}=E I_{L}(A)$ which cannot be happen for $a_{1} \neq a_{2}$.

Quite similar situation happens for $\lambda_{1}>0, \lambda_{2}>$ 0 and $\lambda_{3}=0$ since then we get $t_{1}=t_{2}=a_{1}=$ $E I_{L}(A)$ which, of course, cannot hold for $a_{1} \neq a_{2}$.

Finally, the last possible case, i.e. $\lambda_{1}>0, \lambda_{2}>0$ and $\lambda_{3}>0$, does not also produce any new solution since it implies $a_{1}=t_{1}=t_{2}=a_{2}$ which contradicts our assumption that $a_{1}<a_{2}$.

Therefore, after discussing all possible cases related to (18)-(26) we have actually proved the following proposition.

Proposition 1 Let $A$ denote a fuzzy number with the support $\left[a_{1}, a_{4}\right]$ and core $\left[a_{2} . a_{3}\right]$. Then the left arm of the trapezoidal fuzzy number $T(A)=$ $T\left(t_{1}, t_{2}, t_{3}, t_{4}\right)$ nearest to fuzzy number $A$ and preserving its expected interval is defined by $t_{1}$ and $t_{2}$ given as follows: 
(a1) if $a_{1}<4 E I_{L}(A)-6 \int_{0}^{1} \alpha A_{L}(\alpha) d \alpha$ and $6 \int_{0}^{1} \alpha A_{L}(\alpha) d \alpha-2 E I_{L}(A)<a_{2}$ then

$$
\begin{aligned}
& t_{1}=4 E I_{L}(A)-6 \int_{0}^{1} \alpha A_{L}(\alpha) d \alpha \\
& t_{2}=6 \int_{0}^{1} \alpha A_{L}(\alpha) d \alpha-2 E I_{L}(A)
\end{aligned}
$$

(b1) if $4 E I_{L}(A)-6 \int_{0}^{1} \alpha A_{L}(\alpha) d \alpha<a_{1}$ and $6 \int_{0}^{1} \alpha A_{L}(\alpha) d \alpha-2 E I_{L}(A)<a_{2}$ then

$$
\begin{aligned}
t_{1} & =a_{1} \\
t_{2} & =2 E I_{L}(A)-a_{1}
\end{aligned}
$$

(c1) if $a_{1}<4 E I_{L}(A)-6 \int_{0}^{1} \alpha A_{L}(\alpha) d \alpha$ and $a_{2}<$ $6 \int_{0}^{1} \alpha A_{L}(\alpha) d \alpha-2 E I_{L}(A)$ then

$$
\begin{aligned}
t_{1} & =2 E I_{L}(A)-a_{2} \\
t_{2} & =a_{2} .
\end{aligned}
$$

(d1) if $4 E I_{L}(A)-6 \int_{0}^{1} \alpha A_{L}(\alpha) d \alpha<a_{1}$ and $a_{2}<$ $6 \int_{0}^{1} \alpha A_{L}(\alpha) d \alpha-2 E I_{L}(A)$ then

$$
\begin{aligned}
& t_{1}=a_{1}, \\
& t_{2}=a_{2} .
\end{aligned}
$$

Looking on this proposition one immediately conclude that the particular solution depends on the relationship between the lower bounds of the support and core of the fuzzy number under study and two values $4 E I_{L}(A)-6 \int_{0}^{1} \alpha A_{L}(\alpha) d \alpha$ and $6 \int_{0}^{1} \alpha A_{L}(\alpha) d \alpha-2 E I_{L}(A)$, respectively. Thus let us try to find a suitable interpretation for these two critical values. It seems that the notion of the left spread $L S P(A)$ of a fuzzy number $A$ might be helpful there. We define $\operatorname{LSP}(A)$ by (see, e.g., ???)

$$
\begin{aligned}
\operatorname{LSP}(A) & =6 \int_{0}^{1}\left(\alpha-\frac{1}{2}\right) A_{L}(\alpha) d \alpha \\
& =6 \int_{0}^{1} \alpha A_{L}(\alpha) d \alpha-3 E I_{L}(A) .
\end{aligned}
$$

By (29) $L S P(A) \geq 0$ for any fuzzy number $A$. It is easily seen that using this notion we can express Proposition 1 in the following equivalent way.

Proposition 2 Let $A$ denote a fuzzy number with the support $\left[a_{1}, a_{4}\right]$ and core $\left[a_{2}, a_{3}\right]$. Then the left arm of the trapezoidal fuzzy number $T(A)=$ $T\left(t_{1}, t_{2}, t_{3}, t_{4}\right)$ nearest to fuzzy number $A$ and preserving its expected interval is defined by $t_{1}$ and $t_{2}$ given as follows:

(a1) if $a_{1}<E I_{L}(A)-L S P(A)$ and $E I_{L}(A)+$ $L S P(A)<a_{2}$ then

$$
\begin{aligned}
& t_{1}=E I_{L}(A)-L S P(A) \\
& t_{2}=E I_{L}(A)+L S P(A)
\end{aligned}
$$

(b1) if $E I_{L}(A)-L S P(A)<a_{1}$ and $E I_{L}(A)+$ $\operatorname{LSP}(A)<a_{2}$ then

$$
\begin{aligned}
t_{1} & =a_{1}, \\
t_{2} & =2 E I_{L}(A)-a_{1}
\end{aligned}
$$

(c1) if $a_{1}<E I_{L}(A)-L S P(A)$ and $a_{2}<E I_{L}(A)+$ $L S P(A)$ then

$$
\begin{aligned}
t_{1} & =2 E I_{L}(A)-a_{2} \\
t_{2} & =a_{2} .
\end{aligned}
$$

(d1) if $E I_{L}(A)-L S P(A)<a_{1}$ and $a_{2}<E I_{L}(A)+$ $\operatorname{LSP}(A)$ then

$$
\begin{aligned}
& t_{1}=a_{1} \\
& t_{2}=a_{2} .
\end{aligned}
$$

In a similar way we can solve the second minimization program devoted to the right arm of a fuzzy number. We apply again the Karush-KuhnTucker theorem for the local minimizer of $f_{2}(\mathbf{t})$ subject to $h_{2}(\mathbf{t})=t_{3}+t_{4}-2 E I_{U}(A)=0$ and $g_{2}(\mathbf{t})=\left[a_{3}-t_{3}, t_{3}-t_{4}, t_{4}-a_{4}\right] \leq 0$. Then there exist the Lagrange multiplier vector $\eta$ and the KarushKuhn-Tucker multiplier $\tau$ such that

$$
\begin{aligned}
D f_{2}(\mathbf{t})+\xi D h_{2}\left(\mathbf{t}^{*}\right)+\tau^{T} D g_{2}(\mathbf{t}) & =\mathbf{0}^{T}, \\
\tau^{T} g_{2}(\mathbf{t}) & =0, \\
\tau & \geq 0 .
\end{aligned}
$$

In our case, after some calculations, we get

$$
\begin{aligned}
& D f_{2}(\mathbf{t})= {\left[\frac{2}{3} t_{3}+\frac{1}{3} t_{4}-2 \int_{0}^{1} \alpha A_{U}(\alpha) d \alpha\right.} \\
& \frac{1}{3} t_{3}+\frac{2}{3} t_{4}+2 \int_{0}^{1} \alpha A_{U}(\alpha) d \alpha \\
&\left.-2 \int_{0}^{1} \alpha A_{U}(\alpha) d \alpha\right] \\
& D h_{2}\left(\mathbf{t}^{*}\right)=[1,1] \\
& D g_{2}(\mathbf{t})=\left[\begin{array}{cc}
-1 & 0 \\
1 & -1 \\
0 & 1
\end{array}\right] \\
& \tau^{T}=\left[\lambda_{1}, \lambda_{2}, \lambda_{3}\right] .
\end{aligned}
$$

After considering different situations and making some calculations, similarly as for the left arm of a fuzzy number, we obtain a final result. To express it nicely we utilize the upper spread $U S P(A)$ of a fuzzy number $A$ defined by (see, e.g., ???)

$$
\begin{aligned}
U S P(A) & =6 \int_{0}^{1}\left(\frac{1}{2}-\alpha\right) A_{U}(\alpha) d \alpha \\
& =3 E I_{U}(A)-6 \int_{0}^{1} \alpha A_{U}(\alpha) d \alpha
\end{aligned}
$$

It can be shown that $U S P(A) \geq 0$ for any fuzzy number $A$. Finally we obtain the following proposition. 
Proposition 3 Let A denote a fuzzy number with the support $\left[a_{1}, a_{4}\right]$ and core $\left[a_{2} . a_{3}\right]$. Then the right arm of the trapezoidal fuzzy number $T(A)=$ $T\left(t_{1}, t_{2}, t_{3}, t_{4}\right)$ nearest to fuzzy number $A$ and preserving its expected interval is defined by $t_{3}$ and $t_{4}$ given as follows:

(a2) if $a_{3}<E I_{U}(A)-U S P(A)$ and $E I_{U}(A)+$ $U S P(A)<a_{4}$ then

$$
\begin{aligned}
& t_{3}=E I_{U}(A)-U S P(A) \\
& t_{4}=E I_{U}(A)+\operatorname{USP}(A)
\end{aligned}
$$

(b2) if $E I_{U}(A)-U S P(A)<a_{3}$ and $E I_{L}(A)+$ $U S P(A)<a_{4}$ then

$$
\begin{aligned}
t_{3} & =a_{3}, \\
t_{4} & =2 E I_{U}(A)-a_{3}
\end{aligned}
$$

(c2) if $a_{3}<E I_{U}(A)-U S P(A)$ and $a_{4}<E I_{L}(A)+$ $U S P(A)$ then

$$
\begin{aligned}
t_{3} & =2 E I_{U}(A)-a_{4} \\
t_{4} & =a_{4} .
\end{aligned}
$$

(d2) if $E I_{U}(A)-U S P(A)<a_{3}$ and $a_{4}<E I_{L}(A)+$ $U S P(A)$ then

$$
\begin{aligned}
& t_{3}=a_{3} \\
& t_{4}=a_{4} .
\end{aligned}
$$

\section{Discussion}

First of all let us go back to the example which formed a starting point to our considerations.

\section{Example (cont.)}

One can easily compute basic characteristics of the fuzzy number under study required for choosing the proper trapezoidal approximation. Namely, $E I_{L}(A)=-\frac{1}{3}, E I_{U}(A)=14, L S P(A)=\frac{2}{5}$ and $U S P(A)=\frac{78}{5}$.

Since $E I_{L}(A)-\operatorname{LSP}(A)=-\frac{11}{15}>a_{1}=-1$ and $E I_{L}(A)+L S P(A)=\frac{1}{15}>a_{2}=0$, hence, by Prop. 2 we get $t_{1}=2 E I_{L}(A)-a_{2}=-\frac{2}{3}$ and $t_{2}=a_{2}=$ 0 . Moreover, since $E I_{U}(A)-U S P(A)=-\frac{8}{5}<$ $a_{3}=1$ and $E I_{U}(A)+U S P(A)=\frac{148}{5}<a_{4}=40$, then, by Prop. 3 we have $t_{3}=a_{3}=1$ and $t_{4}=$ $2 E I_{U}(A)-a_{3}=27$. Therefore, finally we obtain $T(A)=\left(-\frac{2}{3}, 0,1,27\right)$.

It is easily seen that $\operatorname{core}(A)=\operatorname{core}(T(A))$ and $\operatorname{supp}(T(A)) \subset \operatorname{supp}(A)$.

Let us also notice that our previous operator $T_{1}$ coincides with the approximation operator obtained when $a_{1}<E I_{L}(A)-L S P(A), E I_{L}(A)+$ $L S P(A)<a_{2}, a_{3}<E I_{U}(A)-U S P(A)$ and $E I_{U}(A)+U S P(A)<a_{4}$.

Since all the operators given by Prop. 2 and Prop. 3 preserve the expected interval they satisfy many desired properties described and discussed thoroughly in the literature (see, e.g. [17], [18]).

\section{Conclusion}

In the present contribution we have considered the problem of trapezoidal approximation of fuzzy numbers for that not only preserves the expected interval but satisfy also some natural requirements for the support and core. Due to these improvements we avoid possible troubles with the interpretation of the solution for any fuzzy numbers even those with a very skew membership function.

\section{References}

[1] S. Abbasbandy, B. Asady, The nearest approximation of a fuzzy quantity in parametric form, Appl. Math. Comput. 172 (2006) 624-632.

[2] S. Abbasbandy, E. Ahmady and N. Ahmady, Triangular approximations of fuzzy numbers using $\alpha$-weighted valuations, Soft Comput. 14 (2010) 71-79.

[3] S. Abbasbandy, M. Amirfakhrian, The nearest trapezoidal form of a generalized LR fuzzy number, Int. J. Approx. Reason., 43 (2006) 166-178.

[4] S. Abbasbandy, T. Hajjari, Weighted trapezoidal approximation-preserving cores of a fuzzy number, Comput. Math. Appl. 59 (2010) 30663077 .

[5] A.I. Ban, Approximation of fuzzy numbers by trapezoidal fuzzy numbers preserving the expected interval, Fuzzy Sets Syst. 159 (2008) 1327-1344.

[6] A.I. Ban, Trapezoidal approximations of intuitionistic fuzzy numbers expressed by value, ambiguity, width and weighted expected value, Notes on Intuitionistic Fuzzy Sets 14 (2008) 3847.

[7] A.I. Ban, On the nearest parametric approximation of a fuzzy number-Revisited, Fuzzy Sets Syst. 166 (2009) 3027-3047.

[8] A.I. Ban, Remarks and corrections to the triangular approximations of fuzzy numbers using $\alpha$ weighted valuations, Soft Comput. (2010), doi: 10.1007/s00500-010-0620-0.

[9] S. Chanas, On the interval approximation of a fuzzy number, Fuzzy Sets and Syst. 122 (2001), 353-356.

[10] M. Delgado, M.A. Vila, W. Voxman, On a canonical representation of a fuzzy number, Fuzzy Sets and Syst. 93 (1998), 125-135.

[11] D. Dubois, H. Prade, Operations on fuzzy numbers, Int. J. Syst. Sci. 9 (1978), 613-626.

[12] D. Dubois, H. Prade, The mean value of a fuzzy number, Fuzzy Sets Syst. 24 (1987) 279-300.

[13] P. Grzegorzewski, Metrics and orders in space of fuzzy numbers, Fuzzy Sets Syst. 97 (1998) 8394.

[14] P. Grzegorzewski, Nearest interval approximation of a fuzzy number, Fuzzy Sets Syst. 130 (2002) 321-330. 
[15] P. Grzegorzewski, Trapezoidal approximations of fuzzy numbers preserving the expected interval - algorithms and properties, Fuzzy Sets Syst. 159 (2008) 1354-1364.

[16] P. Grzegorzewski, Algorithms for trapezoidal approximations of fuzzy numbers preserving the expected interval, in: B. Bouchon-Meunier, L. Magdalena, M. Ojeda-Aciego, J.-L. Verdegay, R.R. Yager (Eds.), Foundations of Reasoning under Uncertainty, Springer, 2010, pp. 85-98.

[17] P. Grzegorzewski, E. Mrówka, Trapezoidal approximations of fuzzy numbers, Fuzzy Sets Syst. 153 (2005) 115-35.

[18] P. Grzegorzewski, E. Mrówka, Trapezoidal approximations of fuzzy numbers - revisited, Fuzzy Sets Syst. 158 (2007) 757-768.

[19] P. Grzegorzewski, K. Pasternak-Winiarska, Weighted trapezoidal approximations of fuzzy numbers, in: J.P. Carvalho, D. Dubois, U. Kaymak, J.M.C. Sousa (Eds.), Proceedings of the Joint 2009 International Fuzzy Systems Association World Congress and 2009 European Society of Fuzzy Logic and Technology Conference, Lisbon, 2009, pp. 1531-1534.

[20] P. Grzegorzewski, K. Pasternak-Winiarska, Bisymmetrically weighted trapezoidal approximations of fuzzy numbers, in: A. Abraham, J.M. Benitez Sanchez, F. Herrera, V. Loia, F. Marcelloni, S. Senatore (Eds.), Proceedings of Ninth International Conference on Intelligent Systems Design and Applications, Pisa, 2009, pp. 318323.

[21] S. Heilpern, The expected value of a fuzzy number, Fuzzy Sets Syst. 47 (1992) 81-86.

[22] E.N. Nasibov, S. Peker, On the nearest parametric approximation of a fuzzy number, Fuzzy Sets Syst. 159 (2008) 1365-1375.

[23] C.-T. Yeh, Trapezoidal and triangular approximations preserving the expected interval, Fuzzy Sets Syst. 159 (2008) 1345-1353.

[24] C.-T. Yeh, On improving trapezoidal and triangular approximations of fuzzy numbers, Int. J. Approx. Reason. 48 (2008) 297-313.

[25] C.-T. Yeh, Weighted trapezoidal and triangular approximations of fuzzy numbers, Fuzzy Sets Syst. 160 (2009) 3059-3079.

[26] C.-T. Yeh, Weighted semi-trapezoidal approximations of fuzzy numbers, Fuzzy Sets Syst. (2010), doi:10.1016/j.fss.2010.11.001.

[27] W. Zeng, H. Li, Weighted triangular approximation of fuzzy numbers, Int. J. Approx. Reason. 46 (2007) 137-150. 